\title{
p53 mutant breast cancer patients expressing p53 $\gamma$ have as good a prognosis as wild-type p53 breast cancer patients
}

Jean-Christophe Bourdon ${ }^{1,2^{*}+}$, Marie P Khoury ${ }^{1,2,3+}$, Alexandra Diot ${ }^{1}$, Lee Baker ${ }^{1}$, Kenneth Fernandes ${ }^{1}$, Mustapha Aoubala', Philip Quinlan', Colin A Purdie ${ }^{4}$, Lee B Jordan ${ }^{4}$, Anne-Catherine Prats ${ }^{1,2,3}$, David P Lane ${ }^{5}$, Alastair M Thompson ${ }^{1,6}$

\begin{abstract}
Introduction: Normal function of the p53 network is lost in most cancers, often through p53 mutation. The clinical impact of p53 mutations in breast cancer remains uncertain, especially where p53 isoforms may modify the effects of these p53 mutations.

Methods: Expression of p53 $\beta$ and p53 $\gamma$ isoforms, the isoforms identified in normal breast tissue, was detected by reverse transcription polymerase chain reaction from a cohort of 127 primary breast tumours. Expression of p53 $\beta$ and $\mathrm{p} 53 \gamma$ isoforms was analysed in relation to clinical markers and clinical outcomes (5 years) by binary logistic regression, Cox proportional hazards regression and Kaplan-Meier survival analyses.

Results: $\mathrm{p} 53 \beta$ and $\mathrm{p} 53 \gamma$ were not randomly expressed in breast cancer. $\mathrm{p} 53 \beta$ was associated with tumour oestrogen receptor (ER) expression, and p53 $\gamma$ was associated with mutation of the p53 gene. The patient group with the mutant $p 53$ breast tumour-expressing p53 $\gamma$ isoform had low cancer recurrence and an overall survival as good as that of patients with wild-type p53 breast cancer. Conversely, patients expressing only mutant p53, without p53 $\gamma$ isoform expression, had a particularly poor prognosis.

Conclusions: The determination of p53 $\gamma$ expression may allow the identification, independently of the ER status, of two subpopulations of mutant p53 breast cancer patients, one expressing p53 $\gamma$ with a prognosis as good as the wild-type $p 53$ breast cancer patients and a second one not expressing p53 $\gamma$ with a particularly poor prognosis. The p53 $\gamma$ isoform may provide an explanation of the hitherto inconsistent relationship between p53 mutation,

treatment response and outcome in breast cancer.
\end{abstract}

\section{Introduction}

The p53 pathway is ubiquitously abnormal in human cancers, either through mutation of the $p 53$ gene or via modification of p53 function by interaction with oncogenic cellular or viral proteins $[1,2]$. Somatic $p 53$ gene mutations, found in about $25 \%$ of breast cancers, are associated with poor prognosis [3,4]. Patients bearing mutant $p 53$ breast cancer have resistance to several chemotherapy agents but may be more sensitive to taxanes, at least in the neoadjuvant setting [5-10]. However, the

\footnotetext{
* Correspondence: j.bourdon@dundee.ac.uk

+ Contributed equally

${ }^{1}$ Centre for Oncology and Molecular Medicine, Inserm-European Associated

Laboratory, University of Dundee, Dundee, DD1 9SY, UK

Full list of author information is available at the end of the article
}

uncertainties around the relationships between $p 53$ mutation, therapeutic response and outcome in breast cancer suggest that additional factors may be involved.

The human $p 53$ gene expresses at least nine different p53 protein isoforms containing different domains of the p53 protein ( $553, \mathrm{p} 53 \beta, \mathrm{p} 53 \gamma, \Delta 133 \mathrm{p} 53 \alpha, \Delta 133 \mathrm{p} 53 \beta$, $\Delta 133 \mathrm{p} 53 \gamma, \Delta 40 \mathrm{p} 53 \alpha, \Delta 40 \mathrm{p} 53 \beta$ and $\Delta 40 \mathrm{p} 53 \gamma)$ as a result of multiple splicing, alternative initiation of translation and internal promoter usage [11-13]. The p53 isoforms are differentially expressed in normal human tissues, with normal breast tissue expressing p $53, \mathrm{p} 53 \beta$ and p53 $\gamma$ [13]. Abnormal expression of p53 isoforms has been identified in several human cancer types [13-19]. We have previously reported that p53 isoforms such as

\section{() Biomed Central}


p53 3 can interact with p53 and modulate p53 tumour suppressor activity $[13,19,20]$. Taken together, these findings suggest that the p53 isoforms may play a role in human cancers.

In this report, expression of the p53 $\beta$ and $\mathrm{p} 53 \gamma$ isoforms is examined in relation to clinical and pathological markers, p53 mutation and disease outcome in a cohort of 127 randomly selected primary breast tumours. The patient group expressing the p53 $\gamma$ isoform had abrogation of the poor prognostic effect associated with $p 53$ mutation, with a low risk of cancer recurrence and a survival rate as good as that of the patient group bearing wild-type p53 breast cancer. Conversely, patients expressing only mutant $p 53$, without $\mathrm{p} 53 \gamma$ isoform expression, had a particularly poor prognosis. The $\mathrm{p} 53 \gamma$ isoform may explain the inconsistent relationship between $p 53$ mutation and breast cancer in the literature.

\section{Materials and methods Clinical samples}

Previously untreated operable primary breast cancer in 127 Caucasian women (age range, 32 to 89 years; median age, 60 years) with sufficient tumour tissue surplus for diagnostic requirements and complete clinical and pathological data was analysed. Tumour tissues were macrodissected by a specialist breast pathologist and snap-frozen in liquid nitrogen prior to storage at $-80^{\circ} \mathrm{C}$. Samples were examined following Local Research Ethics Committee approval under delegated authority from the Tayside Tissue Bank. The Tayside Tissue Bank has received ethical approval for its activities (REC Reference 07/S1402/90).

\section{Reverse transcription-polymerase chain reaction analysis} Approximately $10 \mathrm{mg}$ of tumour tissue ( $>40 \%$ of tumour cells) was homogenised in $750 \mu \mathrm{L}$ of QIAzol lysis reagent (Qiagen Ltd, Crawley, West Sussex, UK), and total RNA was extracted (Qiagen). RNA quality was assessed using the BioAnalyzer $2100^{\mathrm{TM}}$ (Agilent Technologies, Palo Alto, CA, USA) prior to reverse transcription-polymerase chain reaction (RT-PCR) analysis, and all samples with a $28 \mathrm{~S}: 18 \mathrm{~S}$ ratio $<1.2$ were discarded. RT was performed with $0.5 \mu \mathrm{g}$ of total RNA using the Cloned AMV Reverse Transcription Kit (Invitrogen, Paisley, UK), and cDNA quality was confirmed by PCR amplification of actin. Samples for which actin could not be amplified after 30 cycles of PCR were discarded. p53 isoform cDNA was amplified by two consecutive PCR assays (nested PCR) of 30 cycles each, and the PCR primers used were specific for each of the p53 isoforms analysed. The different primers used and their corresponding sequences are indicated in Table S1 in Additional file 1. To determine $\mathrm{p} 53 \gamma$ mutation status, the entire open reading frame of the isoform was sequenced using the Sanger method (BigDye Terminator, ABI 3730 Genetic Analyser; (Applied Biosystems, Warrington, UK) with the primers JWF (5'-AGCCAAGTCTGTGACTTGCA) and MP9ER (5'-TCTCCCAGGACAGCACAAA CACG).

\section{p53 mutation analysis}

The $p 53$ mutation status was determined using $100 \mathrm{ng}$ of genomic DNA extracted from homogenised frozen tissues as described previously using the AmpliChip p53 Test (Roche Diagnostics, Pleasanton, CA, USA) [21].

\section{Tumour grade, oestrogen receptor, progesterone receptor and HER2 status}

Immunohistochemical staining was carried out on $4-\mu \mathrm{m}$ sections of formalin-fixed, paraffin-embedded tumours with the mouse monoclonal anti-oestrogen receptor $\alpha$ (ER) antibody 6F11 (Novocastra Laboratories Ltd, Newcastle upon Tyne, UK), progesterone receptor (PR) antibody clone 16 (Novocastra Laboratories Ltd) and mouse monoclonal anti-HER2 antibody CB11 (Novocastra Laboratories Ltd). Additional analyses were performed according to histological tumour grade (graded by a specialist consultant breast pathologist); pathological tumour size (pT1, tumours <2 cm; pT2 and pT3 cancers, tumours $\geq 2 \mathrm{~cm}$ ) [22]. ER status (ER negative 0 to 3 versus ER positive 4 to 18 ) was determined using the quickscore method [23]. Briefly, immunoreactivity scored semiquantitatively for both the intensity and the proportion of cells staining. Intensity was given scores from 0 to 3 (no staining $=0$, light staining $=1$, moderate staining $=2$ and strong staining $=3$ ) and proportion was given scores from 1 to $6(0 \%$ to $4 \%=1,5 \%$ to $20 \%=2,21 \%$ to $40 \%=3,41 \%$ to $60 \%=4,61 \%$ to $80 \%=5$ and $81 \%$ to $100 \%=6$ ). The two scores were then multiplied to obtain the final result of 0 to 18 . Human epidermal growth factor receptor 2 (HER2) scoring was performed as previously described [24].

\section{Statistical analysis}

The primary outcomes in this study were breast cancerspecific overall survival (abbreviated to overall survival) and breast cancer-specific disease-free survival (abbreviated to disease-free survival or cancer recurrence throughout the text), and accordingly, non-breast cancer deaths were censored at the time of death (that is, at the time of death, the women were considered to have survived breast cancer but died as a result of other causes). Statistical analysis was performed using Minitab version 15.1.0.0 statistical software (Minitab Inc., PA 16801-3008, USA) for $\chi^{2}$, two-sided Fisher's exact test and Kaplan-Meier analyses. These univariate analyses test for associations between variables in a pairwise 
manner (for example, A versus B), but they do so without adjusting for influences exerted by other associated variables (for example, both $A$ and $B$ may be associated with confounding variables $\mathrm{C}, \mathrm{D}$ and $\mathrm{E}$, casting doubt on the validity of the relationship between $\mathrm{A}$ and $\mathrm{B}$ ).

To clarify the univariate analyses and adjust for possible confounding variables, the selected variables were interrogated using the multivariate methods of binary logistic regression (BLR) with associated odds ratios (OR) and the Cox proportional hazards regression model (CR) with associated risk ratios (RR), both utilising the backwards stepwise elimination method. (For more detailed methods, read the "method" section in Additional file 2.)

In the tables of results for these multivariate analyses, the $\beta$ value is a regression coefficient that indicates the strength of association between the predictor and response variables, where a large $\beta$ indicates a strong association. A positive $\beta$ indicates a positive association between the predictor and response variables, whilst a negative $\beta$ indicates a negative association.

The OR is used to assess the risk of a particular outcome if a certain factor (or exposure) is present, indicating how much more likely it is that someone who is exposed to the factor under study will develop the outcome as compared to someone who is not exposed. If the odds are greater than 1 , then the event is more likely to happen than not, whilst if the odds are less than 1, then the event is less likely. One 'reads' the risk ratios in precisely the same way.

The results of the univariate and multivariate analyses were consistent, and for clarity and brevity only the results of BLR, CR and Kaplan-Meier analyses are presented. Throughout the analyses the null hypothesis was rejected at an $\alpha$ level of $10 \%(P<0.10)$, and observations considered to be marginal (that is, worthy of further analysis) for an $\alpha$ level between 5\% and $10 \%$ $(0.05 \leq P \leq 0.10)$ and significant at $5 \%(P<0.05)$. The $P$ value represents the probability of error that is involved in accepting our observed result as valid. For example, $P=0.05$ indicates that there is a $5 \%$ probability that the relation between the variables found in the sample occurred by chance.

\section{Results \\ p53 $\beta$ and p53 $\gamma$ isoform expression in primary breast cancers}

Cancers from 127 women (median age, 60.0 years; age range, 32.1 to 89.1 years) were examined. The majority of cancers were ductal carcinomas at $84 \%$ (107 of 127). Of these cases, $77 \%$ (98 of 127) were ER-positive, $62 \%$ (79 of 127) were PR-positive, 14\% (17 of 119) were HER2-positive and 22\% (28 of 127) had a tumour containing mutant $p 53$. Approximately 50\% (63 of 127) of the patients had axillary lymph node metastasis; tumours were grade 1 (16 cancers), grade 2 (48 cancers) or grade 3 (61 cancers), respectively. This patient population was therefore representative of symptomatic primary breast cancers in a Western country.

Expression of p53 $\beta$ and p53 $\gamma$ was successfully analysed in the 127 primary breast cancers by using RT-PCR (Figure 1). On testing in triplicate, breast cancers consistently demonstrated p $53 \beta$ expression (36\%; 46 of 127) and p53 $\gamma$ expression (37\%; 47 of 127). Only 19\% (24 of 127) of tumours expressed both $\mathrm{p} 53 \beta$ and $\mathrm{p} 53 \gamma$.

Univariate statistical analysis determined that both p53 $\beta$ and p53 $\gamma$ were associated with clinical markers (data not shown). To clarify these associations and adjust for possible confounding variables, BLR analyses were performed to examine the associations of the various clinical markers with p53 $\beta$ and p53 (Tables S2 and S3 in Additional file 1). p53 $\beta$ isoform expression was independently associated with p53 $\gamma$ expression $(P=0.008$, BLR) (Table 1), and p53 $\gamma$ expression was independently associated with $p 53$ mutation $(P=0.002$, BLR) (Table 1$)$. However, after adjusting for other associated clinical markers, p53 $\beta$ expression was not associated with $p 53$ mutation $(P=0.970, B L R)$ (Table 1$)$. For tumours bearing the p53 mutation, p53 $\gamma$ cDNA was directly sequenced using Sanger methods and was found to contain the same $p 53$ mutation identified by the AmpliChip p53 Test, indicating that $\mathrm{p} 53 \gamma$ was expressed by tumour tissue from the same allele as the $p 53$ mutation and not by stromal tissues. Most $p 53$ mutations were hemizygous missense mutations affecting the DNA-binding domain of p53. Since the $p 53$ gene was mutated at different codons in our cohort of breast cancer, there were not enough cases with the same $p 53$ mutation for the statistical analysis.

\section{p53 $\beta$ and p53 $\gamma$ isoform expression, clinical and pathological associations}

p53 $\beta$ expression was independently associated with ER status $(P=0.033, \mathrm{BLR})$ (Table S4 in Additional file 1 ), but not with PR status. p53 $\gamma$ expression was not associated with either ER or PR status. p53 $\beta$ and p53 $\gamma$ isoform expression was not associated with tumour type, menopausal status, age of cancer onset or HER2 status (data not shown).

As expected, p53 mutation was independently associated with cancer recurrence and death $(P=0.013$ and $P=0.017$, respectively; BLR) (Table 1 ). However, neither $\mathrm{p} 53 \beta$ nor $\mathrm{p} 53 \gamma$ isoform expression was associated with cancer recurrence $(P=0.198$ and $P=0.636$, respectively) (Table 1$)$ or death $(P=0.082$ and $P=0.783$, respectively) (Table 1 ).

To determine whether the associations between $p 53$ mutation and the various clinical markers were different in p $53 \beta$ - or p $53 \gamma$-positive tumours, data were stratified by 


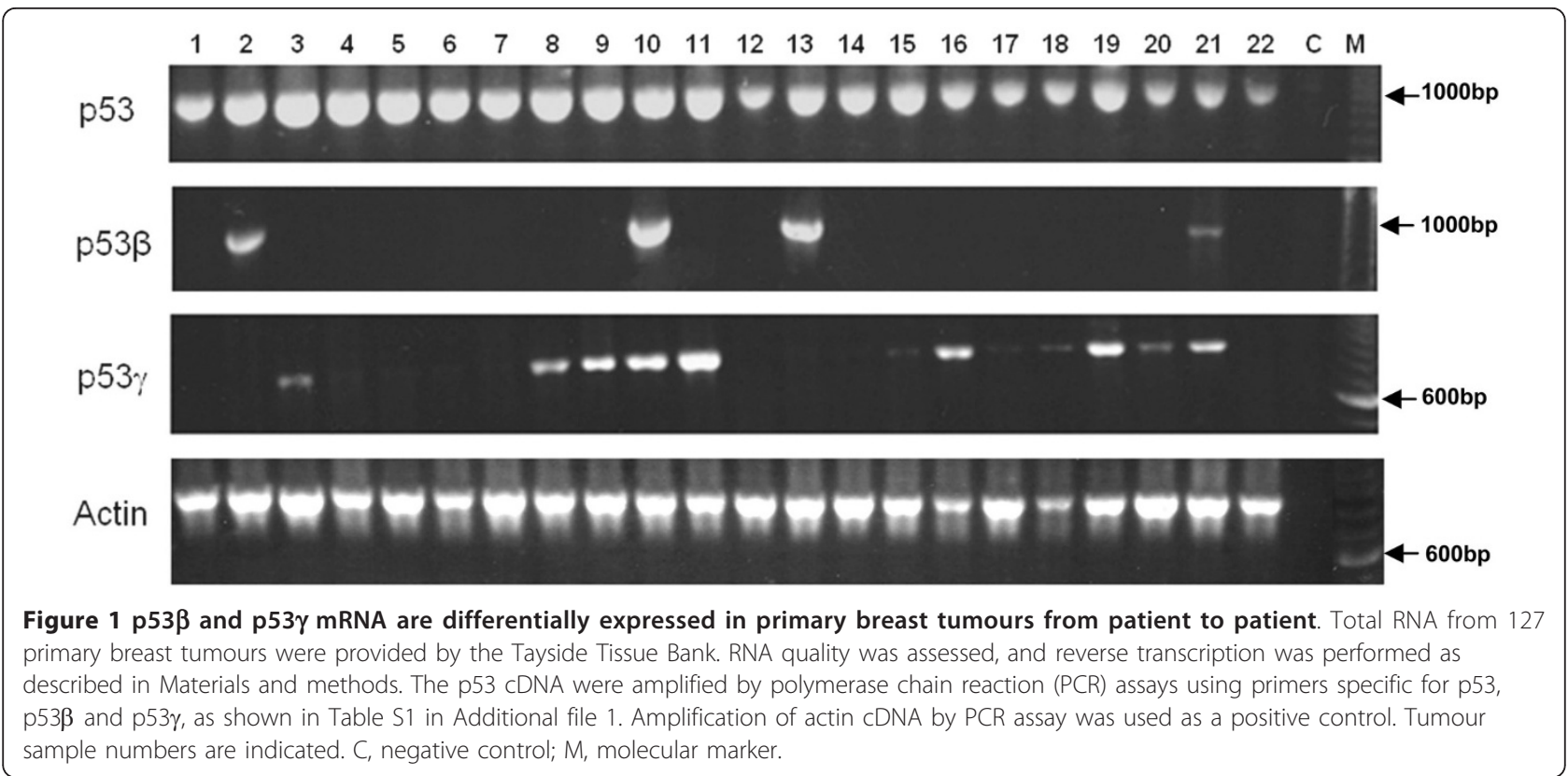

p53 $\beta$ and p53 $\gamma$ expression status. BLR analyses were performed to examine the associations between markers in the p $53 \beta$ - and p $53 \gamma$-positive and p $53 \beta$ - and p $53 \gamma$-negative cohorts (Table 2, and Tables S2 and S3 in Additional file 1).

Regarding p53ß, p53 mutation status was marginally associated with cancer recurrence and death in the p533-negative cohort $(P=0.059$ and $P=0.072$, respectively; BLR) (Table S2 in Additional file 1), while in the p53 $\beta$-positive cohort $p 53$ mutation status was not associated with death but was associated with cancer recurrence $(P=0.018$, BLR; Table S2 in Additional file 1).

Regarding p53 $\gamma$, p53 mutation status was independently associated with cancer recurrence and death in the p53 $\gamma$-negative cohort $(P=0.001$ and $P=0.002$, respectively; BLR) (Table 2, and Table S3 in Additional file 1). Interestingly, $p 53$ mutation status was not associated with cancer recurrence or death in the p53y-positive cohort $(P=0.579$ and $P=0.282$, respectively; BLR) (Table 2, and Table S3 in Additional file 1), despite the greater proportion of grade 3 cancers with $p 53$ mutations $(61.5 \% ; 16$ of 26$)$ in the p53 $\gamma$-positive cohort compared with the p53 $\gamma$-negative cohort (25.7\%; 9 of 35 ). These data suggest that p $53 \gamma$ expression delineates two subpopulations of mutant $p 53$ breast cancer patients with markedly different outcomes.

\section{$p 53 \beta, p 53 \gamma$ and clinical outcome}

To investigate the association between $\mathrm{p} 53 \beta$ or $\mathrm{p} 53 \gamma$ isoform expression and the clinical markers in relation to survival and cancer recurrence, we performed CR analyses that included p $53 \beta$, p $53 \gamma, p 53$ mutation status and clinical markers (Table 3). These analyses demonstrated the expected associations between prognosis (death and cancer recurrence) and ER, PR, tumour grade, $p 53$ mutation, HER2 or lymph node status (Table 3, rows af), but did not show any independent association for p53 $\beta$ or p53 $\gamma$ isoforms (Table 3, rows $g$ and $h$ ).

Further, to determine the degree of interdependence between the variables $\mathrm{p} 53 \beta, \mathrm{p} 53 \gamma$ and $p 53$ mutation with respect to survival and cancer recurrence, we aggregated these variables into combined variables and reran the $C R$ analyses. We thus formed a binary variable (p53m\&p53 $\beta$ ) that was positive when p $53 \beta$ was expressed and the $p 53$ gene was mutated, but negative otherwise. Similarly, we formed a binary variable (p53m\&p53 $)$ that was positive when $\mathrm{p} 53 \gamma$ was expressed and the $p 53$ gene was mutated, but negative otherwise. The results of using such combined variables (Table 3, row $\mathrm{i}$ and row $\mathrm{j}$ ) allowed us to determine that $p 53$ mutation is no longer associated with death or cancer recurrence when $\mathrm{p} 53 \beta$ or $\mathrm{p} 53 \gamma$ is expressed (Table 3, compare row $\mathrm{c}$ with row $\mathrm{i}$ or row $\mathrm{j}$ ). This effect was independent of ER status and therefore independent of endocrine therapy (in this study, all ERpositive patients were treated with tamoxifen $20 \mathrm{mg}$ for 5 years as standard adjuvant therapy). Moreover, there was also no significant difference in the ER status of patients bearing $p 53$ mutations between the p53 $\gamma$-positive and

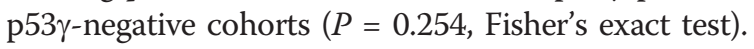

Furthermore, given the association between p $53 \beta$ and p53 $\gamma$ expression (Table 1), we performed CR analysis to determine the combined effects of p53 $\gamma$ and $p 53$ mutation in the absence of $p 53 \beta$ and reciprocally the combined effects of $\mathrm{p} 53 \beta$ and $p 53$ mutation in the absence of $\mathrm{p} 53 \gamma$. We formed a binary variable (p53m\&p53 $\beta^{+}$ 
Table $1 \mathrm{p} 53 \beta$ and $\mathrm{p} 53 \gamma$ expression is associated with clinical markers

\begin{tabular}{|c|c|c|c|c|}
\hline \multirow[b]{2}{*}{$\begin{array}{l}\text { Response } \\
\text { variable }\end{array}$} & \multicolumn{4}{|c|}{ All data } \\
\hline & $\begin{array}{l}\text { Predictor } \\
\text { variable }\end{array}$ & $\beta$ & $\begin{array}{l}P \\
\text { value }\end{array}$ & OR $(95 \% \mathrm{Cl})$ \\
\hline$\overline{p 53 \beta}$ & p53r & 1.01 & 0.008 & 2.75 (1.29 to 5.84$)$ \\
\hline \multirow[t]{2}{*}{ p53r } & p53m & 1.47 & 0.002 & 4.33 (1.74 to 10.78$)$ \\
\hline & $\mathrm{p} 53 \beta$ & 1.01 & 0.012 & 2.74 (1.24 to 6.04$)$ \\
\hline \multirow[t]{5}{*}{ p53m } & $\mathrm{p} 53 \gamma$ & 1.74 & 0.002 & 5.70 (1.92 to 16.93$)$ \\
\hline & $\mathrm{p} 53 \beta$ & -0.02 & 0.970 & $0.98(0.28$ to 3.43$)$ \\
\hline & Tumour grade 3 & 2.85 & $<0.001$ & $\begin{array}{l}17.31 \text { (4.02 to } \\
74.45)\end{array}$ \\
\hline & ER & -2.74 & 0.019 & 0.06 (0.01 to 0.64$)$ \\
\hline & $P R$ & 2.51 & 0.032 & $\begin{array}{l}12.27(1.25 \text { to } \\
120.74)\end{array}$ \\
\hline \multirow[t]{5}{*}{ Overall survival } & p53m & -1.44 & 0.017 & 0.24 (0.07 to 0.78$)$ \\
\hline & $P R$ & 1.48 & 0.014 & 4.41 (1.34 to 14.45$)$ \\
\hline & Tumour size & -1.22 & 0.036 & 0.29 (0.09 to 0.93$)$ \\
\hline & $\mathrm{p} 53 \gamma$ & -0.18 & 0.783 & 0.83 (0.22 to 3.10$)$ \\
\hline & $\mathrm{p} 53 \beta$ & 1.15 & 0.082 & 3.17 (0.87 to 11.60$)$ \\
\hline \multirow{4}{*}{$\begin{array}{l}\text { Cancer } \\
\text { recurrence }\end{array}$} & p53m & 1.25 & 0.013 & 0.29 (0.11 to 0.76$)$ \\
\hline & $P R$ & -1.26 & 0.009 & 3.52 (1.37 to 9.04$)$ \\
\hline & $\mathrm{p} 53 \gamma$ & -0.28 & 0.636 & 1.33 (0.41 to 4.30$)$ \\
\hline & $\mathrm{p} 53 \beta$ & -0.74 & 0.198 & 2.10 (0.68 to 6.47$)$ \\
\hline
\end{tabular}

Variables were analysed using binary logistic regression analysis utilising the backwards stepwise elimination method. Lymph node status, tumour grade, p53 mutation status (p53m), p53 $\beta, p 53 \gamma$, human epidermal growth factor receptor 2 (HER2 or ErbB2), oestrogen receptor (ER) and progesterone receptor (PR) expression were included in the analyses as predictor variables. All independent significant associations between the predictor and response variables were identified (results of run 1). Dependent associations (results of runs $2,3,4$, and so on) are omitted. Only results related to $p 53$ and clinical outcome are presented. Results related to ER/PR, tumour grade and lymph node status are presented in Table S4 in Additional file 1. The $\beta$ coefficients and the odds ratios (OR) with $95 \%$ confidence intervals $(95 \% \mathrm{Cl})$ are indicated.

$\left.\mathrm{p} 53 \gamma^{-}\right)$that was positive when $\mathrm{p} 53 \beta$ was expressed in the absence of $\mathrm{p} 53 \gamma$ expression and the $p 53$ gene was mutated (Table 3, row $\mathrm{k}$ ). We also formed a binary variable ( $\left.\mathrm{p} 53 \mathrm{~m} \& \mathrm{p} 53 \gamma^{+} \mathrm{p} 53 \beta^{-}\right)$that was positive when $\mathrm{p} 53 \gamma$ was expressed in the absence of $\mathrm{p} 53 \beta$ expression and the $p 53$ gene was mutated (Table 3 , row 1 ). These analyses revealed that $p 53$ mutation in patients expressing p $53 \beta$ but not $\mathrm{p} 53 \gamma$ retained the association with death and cancer recurrence (Table 3, compare row c, row i and row $\mathrm{k}$ ), while $p 53$ mutation in patients without p53 $\beta$ but with p53 $\gamma$ was not associated with death and cancer recurrence (Table 3, row c, row $\mathrm{j}$ and row $\mathrm{l}$ ).

Therefore, the apparent abrogation of the association of $p 53$ mutation with poor prognosis in the p53 $\gamma$-positive population (but not in the p53 3 -positive population) indicates that only p $53 \gamma$ allows the identification of a subpopulation of breast cancer patients expressing mutant $p 53$ with a better prognosis than expected.
Table 2 p53 $\gamma$ expression abolishes the association of p53 mutation status with poor prognosis

\begin{tabular}{|c|c|c|c|c|}
\hline \multicolumn{5}{|l|}{ p53\% cohort } \\
\hline $\begin{array}{l}\text { Response } \\
\text { variable }\end{array}$ & $\begin{array}{l}\text { Predictor } \\
\text { variables }\end{array}$ & $\beta$ & $P$ & OR $(95 \% \mathrm{Cl})$ \\
\hline Overall survival & p53m & -2.70 & 0.002 & $\begin{array}{l}0.07 \text { (0.01 to } \\
0.37)\end{array}$ \\
\hline Cancer recurrence & p53m & 2.77 & 0.001 & $\begin{array}{l}0.06 \text { (0.01 to } \\
0.34)\end{array}$ \\
\hline \multicolumn{5}{|l|}{ p53 $\gamma^{+}$cohort } \\
\hline Overall survival & Nothing associated & & & \\
\hline Cancer recurrence & Nothing associated & & & \\
\hline
\end{tabular}

Variables were analysed using binary logistic regression analysis utilising the backwards stepwise elimination method. Lymph node status, tumour grade, $p 53$ mutation status ( $553 \mathrm{~m}), \mathrm{p} 53 \beta, \mathrm{p} 53 \gamma$, human epidermal growth factor receptor 2 (HER2 or ErbB2), oestrogen receptor (ER) and progesterone receptor (PR) expression were included in the analyses as predictor variables. All independent significant associations between the predictor and response variables were identified (results of run 1). Dependent associations (results of runs $2,3,4$, and so on) are omitted. Only results related to p53 and clinical outcomes are presented. Results related to ER/PR, tumour grade and lymph node status are presented in Table S4 in Additional file 1. The $\beta$ coefficient and the odds ratio (OR) with $95 \%$ confidence intervals $(95 \% \mathrm{Cl})$ are indicated.

\section{p53 $\beta$, p53 $\gamma$ expression, p53 mutation and clinical outcomes}

Using Kaplan-Meier log-rank analyses, patients with mutant $p 53$ breast cancer had significantly worse disease-free survival and overall survival than those with wild-type p53 breast cancer (Kaplan-Meier log-rank test, $\chi^{2}=10.51,1 d f, P=0.001$; and $\chi^{2}=6.55,1 d f, P=$ 0.010 , respectively) (Figure S1 in Additional file 3), with a more than three times increased risk of recurrence and death (hazard ratio $(\mathrm{HR})=3.48$ and $\mathrm{HR}=3.16$, respectively). Expression of $\mathrm{p} 53 \beta$ or $\mathrm{p} 53 \gamma$ was not associated with cancer recurrence (Kaplan-Meier log-rank test, $\chi^{2}=0.05,1 d f, P=0.817$; Figure $\mathrm{S} 2$ in Additional file 4, bottom; and $\chi^{2}=0.15,1 d f, P=0.694$; Figure S3 in Additional file 5, bottom, respectively) or with overall survival (Kaplan-Meier log-rank test, $\chi^{2}=0.37,1 d f, P=$ 0.544; Figure S2 in Additional file 4, top; and $\chi^{2}=0.31$, $1 d f, P=0.575$; Figure S3 in Additional file 5, top, respectively).

Patients bearing mutant $p 53$ tumours and expressing the $53 \gamma$ isoform had disease-free survival and overall survival that were not different from patients bearing wild-type p53 tumours, with a low comparative risk of recurrence and a similar risk of death $(\mathrm{HR}=1.72$ and $\mathrm{HR}=1.04$, respectively) (Figure 2A: Kaplan-Meier logrank test, $\chi^{2}=0.76,1 d f, P=0.384$; and Figure $2 \mathrm{~B}$ : Kaplan-Meier log-rank test, $\chi^{2}<0.01,1 d f, P=0.958$, respectively). However, patients bearing mutant $p 53$ tumours without p53 $\gamma$ isoform expression had a high risk of recurrence and subsequent high risk of death $(\mathrm{HR}=7.21$ and $\mathrm{HR}=11.23$, respectively $)$ compared 
Table 3 Cox regression analyses: p53 mutation status in p53 mutant breast cancer patients expressing p53 $\gamma$ is not associated with death and cancer recurrence

\begin{tabular}{|c|c|c|c|c|c|c|c|c|c|c|}
\hline \multirow[b]{2}{*}{ ID } & \multirow[b]{2}{*}{ Run } & \multicolumn{4}{|c|}{ Death } & \multicolumn{5}{|c|}{ Recurrence } \\
\hline & & Predictor & $\beta$ & $P$ & RR (95\% Cl) & Run & Predictor & $\beta$ & $P$ & RR $(95 \%$ Cl) \\
\hline a & 1 & Tumour grade 3 & 2.12 & 0.005 & 8.33 (1.90 to 36.47$)$ & 1 & $P R$ & -0.99 & 0.021 & 0.37 (0.16 to 0.86$)$ \\
\hline$b$ & 2 & PR & -1.36 & 0.011 & 0.26 (0.09 to 0.74$)$ & 1 & p53m & 1.06 & 0.012 & 2.87 (1.27 to 6.51$)$ \\
\hline c & 2 & p53m & 1.1 & 0.026 & 3.00 (1.14 to 7.85$)$ & 2 & Tumour grade 3 & 1.51 & 0.003 & $4.52(1.60$ to 12.11$)$ \\
\hline$d$ & 3 & ER & -1.54 & 0.002 & 0.21 (0.08 to 0.55$)$ & 3 & HER2 & 1.16 & 0.012 & 3.18 (1.29 to 7.83$)$ \\
\hline e & 4 & HER2 & 1.21 & 0.026 & 3.35 (1.16 to 9.65$)$ & 4 & ER & -1.05 & 0.012 & 0.35 (0.16 to 0.79 ) \\
\hline$f$ & $5^{\mathrm{b}}$ & Lymph nodes & 1.19 & 0.037 & 3.30 (1.08 to 10.13$)$ & $5^{\mathrm{b}}$ & Lymph nodes & 0.93 & 0.039 & 2.53 (1.05 to 6.09$)$ \\
\hline g & $6^{\mathrm{b}}$ & p53 $\beta$ & -0.38 & 0.491 & 0.69 (0.24 to 2.00$)$ & $6^{\mathrm{b}}$ & $\mathrm{p} 53 \beta$ & -0.17 & 0.699 & 0.84 (0.35 to 2.03) \\
\hline h & $6^{b}$ & $\mathrm{p} 53 \gamma$ & 0.48 & 0.337 & 1.61 (0.61 to 4.29$)$ & $6^{b}$ & $\mathrm{p} 53 \gamma$ & 0.19 & 0.652 & 1.21 (0.52 to 2.81$)$ \\
\hline i & $5^{b}$ & p53m\&p53 $\beta^{+}$ & 0.07 & 0.451 & 1.47 (0.54 to 3.99) & $5^{b}$ & p53m\&p53 $\beta^{+}$ & 0.97 & 0.053 & 2.65 (0.99 to 7.10$)$ \\
\hline j & $5^{b}$ & p53m\&p53 $\gamma^{+}$ & 0.31 & 0.626 & 1.36 (0.39 to 4.75$)$ & $5^{b}$ & p53m\&p53 $\gamma^{+}$ & 0.49 & 0.333 & 1.63 (0.61 to 4.36$)$ \\
\hline k & 2 & $\mathrm{p} 53 \mathrm{~m} \& \mathrm{p} 53 \beta^{+} \mathrm{p} 53 \gamma^{-}$ & 2.87 & 0.017 & 17.56 (1.66 to 186.15$)$ & 4 & $\mathrm{p} 53 \mathrm{~m} \& \mathrm{p} 53 \beta^{+} \& \mathrm{p} 53 \gamma^{-}$ & 1.71 & 0.021 & 5.51 (1.29 to 23.57$)$ \\
\hline 1 & $5^{\mathrm{b}}$ & $\mathrm{p} 53 \mathrm{~m} \& \mathrm{p} 53 \gamma^{+} \& \mathrm{p} 53 \beta^{-}$ & 0.78 & 0.298 & 2.19 (0.50 to 9.59) & $5^{\mathrm{b}}$ & p53m\&p53 $\gamma^{+} \& p 53 \beta^{-}$ & 0.31 & 0.679 & $1.36(0.32$ to 5.78$)$ \\
\hline
\end{tabular}

${ }^{a}$ Variables were analysed using Cox proportional hazards regression model utilising the backwards stepwise elimination method. Lymph node status, tumour

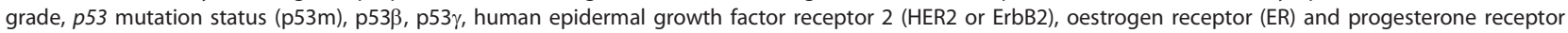
(PR) expression were included in analyses as predictor variables. The run number refers to the run in which the predictor variable was deemed to be statistically significantly associated with the response variable (death or recurrence) and thereafter was excluded from further runs. Rows a-f refer to the statistically significant results in the first iteration of analyses without interaction variables. Of the variables that were not associated with the response variables, $\mathrm{p} 53 \beta$ and $\mathrm{p} 53 \gamma$ are included in the table (rows $\mathrm{g}$ and $\mathrm{h}$ ), but others are omitted for clarity and brevity. Rows i-I refer to results of Cox regression analyses for the interaction predictor variables shown in the presence of all the above predictor variables (data not shown for clarity and brevity). The $\beta$ coefficient and the risk ratio (RR) with $95 \%$ confidence intervals $(95 \% \mathrm{Cl})$ are indicated; ${ }^{b}$ Final run in a given set of analyses.

with patients bearing wild-type p53 tumours (Figure 2; Kaplan-Meier log-rank test: $\chi^{2}=18.33,1 d f, P<0.001$; and $\chi^{2}=20.70,1 d f, P<0.001$, respectively). Consistent with the CR analysis, the Kaplan-Meier log-rank analyses indicate that $\mathrm{p} 53 \gamma$ allows the identification of a subpopulation of breast cancer patients expressing mutant $p 53$ with a better prognosis than expected.

\section{Discussion}

The p53 network is thought to be ubiquitously altered in human cancers, either through mutation of the $p 53$ gene or through inactivation of p53 protein [1]. In breast cancer, it has been difficult to link p53 mutation status to therapeutic response and clinical outcome, suggesting that additional factors may affect the p53 network. We previously reported that the $p 53$ gene expresses at least nine p53 protein isoforms in normal human tissue, including $\mathrm{p} 53 \beta$ and $\mathrm{p} 53 \gamma$, which are differentially expressed in breast cancer as in other types of cancer [13-19]. In this study, we report the analysis of expression of $\mathrm{p} 53 \beta$ and $\mathrm{p} 53 \gamma$ in relation to clinical and pathological markers and disease outcome in a cohort of 127 randomly selected primary breast tumours.

In our cohort, p53 3 expression was detected in $36 \%$ of the primary breast tumours and was associated with ER expression but not with disease outcome. p53 $\gamma$ expression was detected in $37 \%$ of primary breast tumours and was associated with $p 53$ gene mutation. The potentially clinically significant finding was that $\mathrm{p} 53 \gamma$ expression allowed discrimination between two subpopulations of patients bearing mutant $p 53$ tumours: (1) patients bearing mutant $p 53$ cancer and expressing p53 $\gamma$ who had disease-free survival and overall survival as good as patients with wild-type p53 and (2) patients bearing mutant $p 53$ tumours without detectable p53 $\gamma$ isoform expression who had a particularly poor prognosis. Importantly, there was no significant difference in the ER status of patients bearing p53 mutations between the p53 $\gamma$-positive and p53 $\gamma$-negative cohorts $(P=0.254$, Fisher's exact test). Therefore, the better outcomes of the breast cancer patients expressing p53 $\gamma$ and mutant p53 are not due to endocrine therapy in ER-positive cancers.

We have chosen to perform this analysis without previously classifying tumours according to immunohistochemical phenotype (luminal (A and B), HER2, basal (triple-negative: $\mathrm{ER}^{-}, \mathrm{PR}^{-}, \mathrm{HER}^{-}$) and unclassified) because, in our cohort, the low number of tumours in each immunohistochemical phenotype did not allow us to perform CR and Kaplan-Meier log-rank analyses to investigate $\mathrm{p} 53$ isoform expression in relation to clinical outcome. Indeed, among the 85 luminal tumours $\left(\mathrm{ER}^{+}\right.$, $\mathrm{PR}^{+}$and $\mathrm{HER}^{-}$), only 10 tumours expressed mutant $p 53$. This low number of $p 53$ mutations did not allow us to find a significant statistical association between p53 isoform expression and p53 mutation. By contrast, 
A)

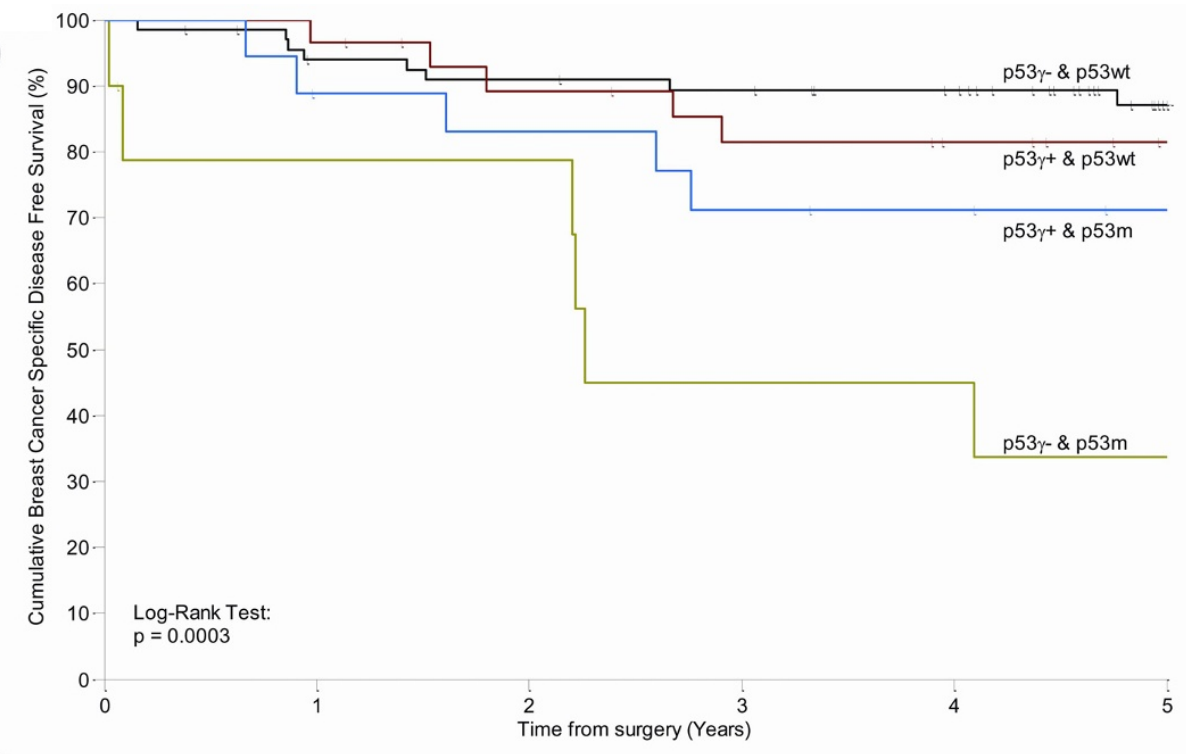

Number At Risk

p53 $\gamma-\&$ p $53 w t$

p53 + \& p $53 w t$

p53 $\gamma$ - \& $p 53 m$

p $53 \gamma+\& p 53 m$

B)

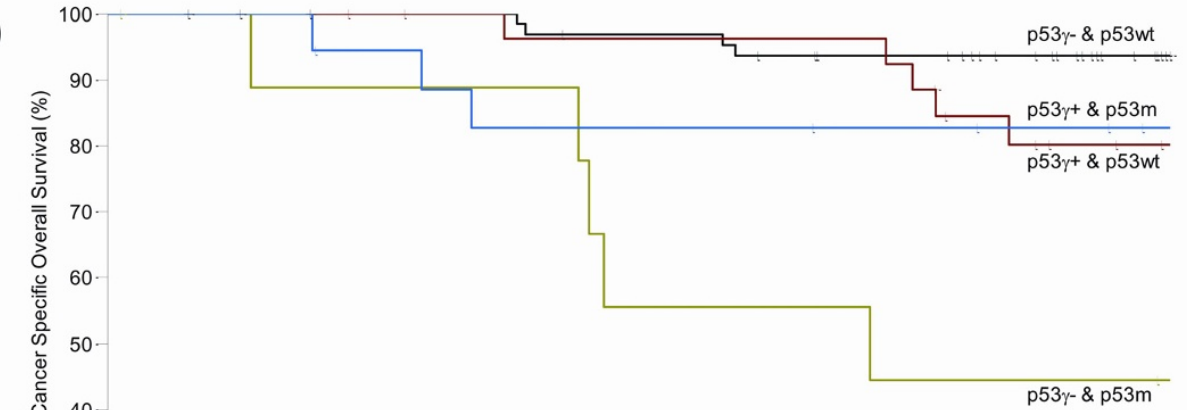

Number At Risk

\begin{tabular}{|c|c|}
\hline p53\%- \& p53wt & 66 \\
\hline$p 53 \gamma+\& p 53 w t$ & 28 \\
\hline p53 $\gamma-\&$ p53m & 10 \\
\hline $\mathrm{p} 53 \gamma+\& \mathrm{p} 53 \mathrm{~m}$ & 18 \\
\hline
\end{tabular}

63

28
$61 \quad 59$

28

7

7

14

57

21

4

12

p53 $\gamma-\& p 53 m$

Figure 2 p53 mutant breast cancer patients expressing p53 $\gamma$ have disease-free survival and overall survival comparable to that of patients bearing wild-type (wt) p53 breast cancer. Nonparametric Kaplan-Meier plots for p53 $\gamma$ expression and p53 gene mutation of (A) disease-free survival $(n=125)$ and $(\mathbf{B})$ overall survival $(n=122)$. Censored cases are indicated by 'I' on the curves. $P$ values are indicated. 
regarding patients who were not in the luminal group (basal and unclassified tumours), 13 of 16 tumours with a p53 mutation expressed p53 $\gamma$ (81\%), whilst 14 of 18 tumours with wild-type $p 53$ did not express p53 $\gamma$ (78\%). This finding confirms that $\mathrm{p} 53 \gamma$ expression is associated with p53 mutation status. Regarding patients who were in the basal group (triple-negative), there was a significant positive association between $553 \gamma$ expression and p53 mutation, with 6 of 7 tumours with a p53 mutation expressing p53 $\gamma(86 \%)$, whilst 9 of 10 tumours with wild-type $p 53$ did not express p53 $\gamma$ (90\%). The result in nonluminal patients is consistent with the results obtained without classifying tumours according to immunohistochemical phenotype. Of note, the lack of association in luminal patients between p53 $\gamma$ expression and $p 53$ mutation is probably due to the low number of p53 mutations in this breast cancer subtype.

By sequencing p53 $\gamma$ cDNA in breast tumours expressing mutant $p 53$, we noted that p53 $\gamma$ cDNA contained the same mutation as the $p 53$ gene, indicating that $\mathrm{p} 53 \gamma$ was expressed by the tumour cells and not by cells from the stroma. Therefore, this finding suggests either that the mutant $\mathrm{p} 53 \gamma$ isoform has an intrinsic activity abrogating the poor prognosis associated with $p 53$ mutation or that $\mathrm{p} 53 \gamma$ is just an inactive marker of better outcomes for mutant $p 53$ breast cancer patients. Future investigations will seek to determine the biological and biochemical activities of mutant p53 $\gamma$ and its interplay with mutant p53 in tumour cells.

We did not differentiate between the different categories of p53 mutations (nonsense mutations, missense mutations, 'DNA-contact' mutations or 'conformational' mutations), as there were not enough cases in each p53 mutation category for confident statistical analysis. However, in larger breast cancer cohorts, it would be interesting to take the different p53 mutation categories and molecular subtypes of breast cancer into account to refine the statistical analysis.

Currently, p53 $\gamma$ expression can be specifically detected only by PCR. From a clinical utility perspective, it would be useful to analyse p $53 \beta$ and p $53 \gamma$ expression by using immunohistochemistry. The mouse monoclonal antibodies DO- 1 and DO-7 recognise p53, p53 $\beta$ and p53 $\gamma$, but not the other p53 isoforms. The rabbit or sheep polyclonal p53 antibodies (CM1 and Sapu, respectively) raised against recombinant full-length human p53 protein recognize all p53 isoforms, while the $\mathrm{KJC} 8$ antibody recognises specifically all p53 $\beta$ isoforms (that is, $\mathrm{p} 53 \beta$, $\Delta 40 \mathrm{p} 53 \beta$ and $\Delta 133 \mathrm{p} 53 \beta)$. However, we have been unable to stain paraffin-embedded sections using the KJC8 antibody. Since p $53 \beta$ and p53 $\gamma$ can be localised in both the nucleus and the cytoplasm, we have attempted to determine by performing immunohistochemistry on paraffin-embedded breast tumour sections using DO-1 or CM1 p53 antibodies whether p53 $\beta$ or p53 $\gamma$ expression is associated with cytoplasmic or nuclear staining. There was no significant association between p53 cytoplasmic or nuclear staining by DO- 1 or CM1 and p53 $\beta$ or $\mathrm{p} 53 \gamma$ expression. Pending the generation of isoformspecific antibodies, p53 immunostaining on tumour sections should be interpreted with caution and should be complemented by PCR analysis to determine p53 isoform mRNA expression in tumours.

Treatment influences were not identified in this analysis, although no taxane, cisplatin or trastuzumab therapy was administered to the patients studied, and anthracycline-based chemotherapy was the standard agent used during the sample accrual period. p53 mutation may be associated with resistance to several chemotherapy agents; but $p 53$ mutant breast cancer may be more sensitive to taxanes, at least in the neoadjuvant setting [5-10], and the predictive value of $p 53$ mutational status in breast cancer remains controversial $[3,4]$. The influence of the $\mathrm{p} 53 \gamma$ isoform in the setting of clinical trials such as the neoadjuvant European Organisation for Research and Treatment of Cancer (EORTC) 10994 Trial, which is testing the association between $p 53$ mutation and taxane versus anthracycline therapy, merits consideration and would provide potential validation of the association of the p53 $\gamma$ isoform with $p 53$ mutation and prognosis in the setting of a randomized, controlled trial. In addition, since mutant $p 53$ cancers are generally of basal or triple-negative phenotype, the influence of the p53 isoforms on platinum therapies and poly (ADP-ribose) polymerase inhibitors in appropriate clinical trials would be of interest. Meanwhile, the apparently dominant effects of the p53 $\gamma$ isoform influencing the p53 network may provide an explanation for the conflicting literature regarding the clinical associations between mutant p53 and breast cancer and issue a warning that clinical decisions made on the basis of $p 53$ mutation status alone may need to be approached with caution.

\section{Conclusions}

In this report, the expression of the p53 $\beta$ and $\mathrm{p} 53 \gamma$ isoforms was examined in relation to clinical and pathological markers, p53 mutation and disease outcome in a cohort of 127 randomly selected primary breast tumours. We determined that p $53 \beta$ and p $53 \gamma$ isoform expression is associated, respectively, with ER status and p53 mutation. p53 $\beta$ or p53 $\gamma$ isoform expression is not independently associated with overall survival or disease-free survival. On the basis of multivariate analyses and Kaplan-Meier analyses, we determined that the breast cancer patient group expressing both mutant $p 53$ and the p53 $\gamma$ isoform has a disease-free and overall survival as good as the patient group bearing wild-type p53 
breast cancer. Conversely, patients expressing only mutant $p 53$ without $\mathrm{p} 53 \gamma$ isoform expression had a particularly poor prognosis. The p53 $\gamma$ isoform may explain the inconsistent relationship between $p 53$ mutation and breast cancer reported in the literature.

\section{Additional material}

Additional file 1: Supplementary Tables. Table S1. Primers used for amplification of p53 isoforms and actin by RT-PCR (nested PCRs). List of the primers used to amplify each human p53 mRNA isoform specifically. Table S2. Binary logistic regression analyses of the p53 $\beta$ positive and p53 $\beta$-negative cohorts. Multivariate analysis of p53 $\beta$ expression in relation to clinical markers and clinical outcomes. Table S3. Binary logistic regression analyses of the $p 53 \gamma$-positive and $p 53 \gamma$ negative cohorts. Multivariate analysis of $p 53 \gamma$ expression in relation to clinical markers and clinical outcomes. Table S4. p53 $\beta$ is associated with oestrogen receptor status. Binary logistic regression analyses including lymph node status, tumour grade, p53 mutation status ( $553 \mathrm{~m})$, p53 $\beta, p 53 \gamma$, HER2 (erbB2), oestrogen receptor (ER) and progesterone receptor (PR) expression as predictor variables.

Additional file 2: Detailed statistical analysis. Explanation of the statistical analysis.

Additional file 3: Figure S1. Analysis of $p 53$ mutation status in relation to breast cancer-specific overall survival and disease-free survival of primary breast cancer patients. Nonparametric Kaplan-Meier plots of (A) disease-free survival (that is, 100\% minus percentage of cancer recurrence) $(n=125)$ and (B) overall survival $(n=122)$ in relation to $p 53$ gene mutation status. Censored cases are shown as 'I' on the curves. $P$ values are based on log-rank tests.

Additional file 4: Figure S2. Analysis of p53ß expression in relation to breast cancer-specific overall survival and disease-free survival of primary breast cancer patients. Nonparametric Kaplan-Meier plots of (A) diseasefree survival (that is, 100\%-percentage of cancer recurrence) $(n=125)$ and (B) overall survival $(n=122)$ in relation to p53 $\beta$ expression. Censored cases are shown as ' $I$ ' on the curves. $P$ values are based on log-rank tests.

Additional file 5: Figure S3. Analysis of p53 $\gamma$ expression in relation to breast cancer-specific overall survival and disease-free survival of primary breast cancer patients. Nonparametric Kaplan-Meier plots of (A) disease free survival (that is, 100\%- percentage of cancer recurrence) $(n=125)$ and (B) overall survival $(n=122)$ in relation to p53y expression. Censored cases are shown as ' 1 ' on the curves. $P$ values are based on log-rank tests.

\section{Abbreviations}

BLR: binary logistic regression; Cl: confidence interval; CR: Cox proportional hazards regression model; ER: oestrogen receptor; OR: odds ratio; PR: progesterone receptor; RT-PCR: reverse transcription-polymerase chain reaction; RR: relative risk.

\section{Acknowledgements}

MK is supported by Egide and Association pour La Recherche sur le Cancer. $A D$ and MA are supported by the Breast Cancer Campaign. KF and JCB are supported by Cancer Research UK. PQ, LB and AD are supported by Breast Cancer Research Scotland. p53 mutation analyses were performed on tumour DNA using the Amplichip p53 Test by Nancy Patten and Lin Wu of Roche Diagnostics (Pleasanton, CA, USA). The sponsors of the study had no role in the study design, data collection, data analysis, data interpretation or the writing of the report. The corresponding authors had full access to all the data in the study and had final responsibility for the decision to submit the report for publication.

\section{Author details}

${ }^{1}$ Centre for Oncology and Molecular Medicine, Inserm-European Associated Laboratory, University of Dundee, Dundee, DD1 95Y, UK. ${ }^{2}$ Institut National de la Santé et de la Recherche Médicale (Inserm), U858, F-31432 Toulouse, France. ${ }^{3}$ Université de Toulouse, UPS, Institute of Molecular Medicine of Rangueil, IFR150, F-31432 Toulouse, France. ${ }^{4}$ Department of Pathology, Ninewells Hospital and Medical School, Dundee, DD1 9SY, UK. ${ }^{5}$ p53 Laboratory (p53Lab), 8A Biomedical Grove, \#06-06, Immunos, Singapore 138648, Singapore. ${ }^{6}$ Department of Surgical Oncology, MD Anderson Cancer Centre, 1400 Holcombe Boulevard, Houston, TX 77030, USA.

\section{Authors' contributions}

$J C B, D P L$ and AT conceived and designed the study. CP and $L$ conducted the pathological review. $A D, M K, K F, M A$ and $M L$ performed the experiments, $M K, L B, P Q, A C P, J C B$ and $A T$ contributed to data analysis and interpretation. $L B, M K, K F, J C B$ and $A T$ contributed to the writing of the report.

\section{Competing interests}

The authors declare that they have no competing interests.

Received: 9 May 2010 Revised: 12 November 2010

Accepted: 20 January 2011 Published: 20 January 2011

\section{References}

1. Vousden KH, Lane DP: p53 in health and disease. Nat Rev Mol Cell Biol 2007, 8:275-283.

2. Petitjean A, Achatz MI, Borresen-Dale AL, Hainaut P, Olivier M: TP53 mutations in human cancers: functional selection and impact on cancer prognosis and outcomes. Oncogene 2007, 26:2157-2165.

3. Olivier M, Petitjean A, Marcel V, Pétré A, Mounawar M, Plymoth A, de Fromentel CC, Hainaut P: Recent advances in p53 research: an interdisciplinary perspective. Cancer Gene Ther 2009, 16:1-12.

4. Olivier M, Langerød A, Carrieri P, Bergh J, Klaar S, Eyfjord J, Theillet C, Rodriguez C, Lidereau R, Bièche I, Varley J, Bignon Y, Uhrhammer N, Winqvist R, Jukkola-Vuorinen A, Niederacher D, Kato S, Ishioka C, Hainaut P, Børresen-Dale AL: The clinical value of somatic TP53 gene mutations in 1,794 patients with breast cancer. Clin Cancer Res 2006, 12:1157-1167.

5. Aas T, Børresen AL, Geisler S, Smith-Sørensen B, Johnsen H, Varhaug JE, Akslen LA, Lønning PE: Specific P53 mutations are associated with de novo resistance to doxorubicin in breast cancer patients. Nat Med 1996, 2:811-814.

6. Geisler S, Børresen-Dale AL, Johnsen H, Aas T, Geisler J, Akslen LA, Anker G, Lønning PE: TP53 gene mutations predict the response to neoadjuvant treatment with 5-fluorouracil and mitomycin in locally advanced breast cancer. Clin Cancer Res 2003, 9:5582-5588.

7. Bertheau P, Espié M, Turpin E, Lehmann J, Plassa LF, Varna M, Janin A, de Thé H: TP53 status and response to chemotherapy in breast cancer. Pathobiology 2008, 75:132-139.

8. Bertheau P, Plassa F, Espié M, Turpin E, de Roquancourt A, Marty M, Lerebours F, Beuzard $Y$, Janin A, de Thé H: Effect of mutated TP53 on response of advanced breast cancers to high-dose chemotherapy. Lancet 2002, 360:852-854.

9. Bertheau $P$, Turpin E, Rickman DS, Espié $M$, de Reyniès $A$, Feugeas JP, Plassa LF, Soliman H, Varna M, de Roquancourt A, Lehmann-Che J, Beuzard Y, Marty M, Misset JL, Janin A, de Thé H: Exquisite sensitivity of TP53 mutant and basal breast cancers to a dose-dense epirubicincyclophosphamide regimen. PLoS Med 2007, 4:e90.

10. Kandioler-Eckersberger D, Ludwig C, Rudas M, Kappel S, Janschek E, Wenzel C, Schlagbauer-Wadl H, Mittlböck M, Gnant M, Steger G, Jakesz R: TP53 mutation and p53 overexpression for prediction of response to neoadjuvant treatment in breast cancer patients. Clin Cancer Res 2000, 6:50-56.

11. Courtois S, Verhaegh G, North S, Luciani MG, Lassus P, Hibner U, Oren M, Hainaut P: $\Delta N-p 53$, a natural isoform of $p 53$ lacking the first transactivation domain, counteracts growth suppression by wild-type p53. Oncogene 2002, 21:6722-6728.

12. Yin Y, Stephen CW, Luciani MG, Fahraeus R: p53 stability and activity is regulated by Mdm2-mediated induction of alternative p53 translation products. Nat Cell Biol 2002, 4:462-467.

13. Bourdon JC, Fernandes K, Murray-Zmijewski F, Liu G, Diot A, Xirodimas DP Saville MK, Lane DP: p53 isoforms can regulate p53 transcriptional activity. Genes Dev 2005, 19:2122-2137.

14. Avery-Kiejda KA, Zhang XD, Adams LJ, Scott RJ, Vojtesek B, Lane DP, Hersey P: Small molecular weight variants of $\mathrm{p} 53$ are expressed in 
human melanoma cells and are induced by the DNA-damaging agent cisplatin. Clin Cancer Res 2008, 14:1659-1668.

15. Anensen N, Oyan AM, Bourdon JC, Kalland KH, Bruserud O, Gjertsen BT: A distinct p53 protein isoform signature reflects the onset of induction chemotherapy for acute myeloid leukemia. Clin Cancer Res 2006, 12:3985-3992.

16. Boldrup L, Bourdon JC, Coates PJ, Sjostrom B, Nylander K: Expression of p53 isoforms in squamous cell carcinoma of the head and neck. Eur $J$ Cancer 2007, 43:617-623.

17. Song W, Huo SW, Lü JJ, Liu Z, Fang XL, Jin XB, Yuan MZ: Expression of p53 isoforms in renal cell carcinoma. Chin Med J (Engl) 2009, 122:921-926.

18. Marabese M, Marchini S, Marrazzo E, Mariani P, Cattaneo D, Fossati R, Compagnoni A, Signorelli M, Moll UM, Codegoni AM, Broggini M: Expression levels of p53 and p73 isoforms in stage I and stage III ovarian cancer. Eur J Cancer 2008, 44:131-141.

19. Fujita K, Mondal AM, Horikawa I, Nguyen GH, Kumamoto K, Sohn JJ, Bowman ED, Mathe EA, Schetter AJ, Pine SR, Ji H, Vojtesek B, Bourdon JC, Lane DP, Harris CC: p53 isoforms $\triangle 133 p 53$ and $p 53 \beta$ are endogenous regulators of replicative cellular senescence. Nat Cell Biol 2009, 11:1135-1142.

20. Chen J, Ng SM, Chang C, Zhang Z, Bourdon JC, Lane DP, Peng J: p53 isoform $\triangle 113 p 53$ is a p53 target gene that antagonizes p53 apoptotic activity via BclxL activation in zebrafish. Genes Dev 2009, 23:278-290.

21. Baker L, Quinlan PR, Patten N, Ashfield A, Birse-Stewart-Bell L, McCowan C, Bourdon JC, Purdie CA, Jordan LB, Dewar JA, Wu L, Thompson AM: p53 mutation, deprivation and poor prognosis in primary breast cancer. $\mathrm{Br} J$ Cancer 2010, 102:719-726.

22. Bloom HJ, Richardson WW: Histological grading and prognosis in breast cancer: a study of 1409 cases of which 359 have been followed for 15 years. Br J Cancer 1957, 11:359-377.

23. Detre S, Saclani Jotti G, Dowsett M: A "quickscore" method for immunohistochemical semiquantitation: validation for oestrogen receptor in breast carcinomas. J Clin Pathol 1995, 48:876-878.

24. Purdie CA, Jordan LB, McCullough JB, Edwards SL, Cunningham J, Walsh M, Grant A, Pratt N, Thompson AM: HER2 assessment on core biopsy specimens using monoclonal antibody CB11 accurately determines HER2 status in breast carcinoma. Histopathology 2010, 56:702-707.

doi:10.1186/bcr2811

Cite this article as: Bourdon et al:: p53 mutant breast cancer patients expressing $\mathrm{p} 53 \gamma$ have as good a prognosis as wild-type p53 breast cancer patients. Breast Cancer Research 2011 13:R7.

\section{Submit your next manuscript to BioMed Central and take full advantage of:}

- Convenient online submission

- Thorough peer review

- No space constraints or color figure charges

- Immediate publication on acceptance

- Inclusion in PubMed, CAS, Scopus and Google Scholar

- Research which is freely available for redistribution

Submit your manuscript at www.biomedcentral.com/submit
Biomed Central 